\title{
Konkordanz zur Inschrift der Acta Severiana
}

Die Konkordanz zeigt eine Gegenüberstellung von neuer Zeilenzählung (linke Spalte, Z. I-3 17) und der Nummerierung in der Edition von Pighi (rechte Spalte; Zählung nach Fragmentgruppen).

\begin{tabular}{|c|c|c|}
\hline $\mathrm{I}=\mathrm{I} \mathrm{I}$ & $43=I_{43}$ & $8_{3}=\mathrm{II} 26$ \\
\hline $2=\mathrm{I} 2$ & $44=\mathrm{I} 44$ & $84=$ II 27 \\
\hline $3=I_{3}$ & $45=I_{45}$ & $85=$ II 28 \\
\hline $4=I_{4}$ & $46=I_{46}$ & $86=$ II 29 \\
\hline$s=I s$ & $47=\mathrm{I} 47$ & \\
\hline $6=\mathrm{I} 6$ & $4^{8}=\mathrm{I} 4^{8}$ & $87=$ III I \\
\hline $7=I_{7}$ & $49=\mathrm{I} 49$ & $88=$ III 2 \\
\hline $8=\mathrm{I} 8$ & $50=$ I 50 & $89=$ III 3 \\
\hline $9=\mathrm{I} 9$ & $S \mathrm{I}=\mathrm{I} S \mathrm{I}$ & $90=$ III 4 \\
\hline $10=$ I 10 & $5^{2}=\mathrm{I} \varsigma^{2}$ & $9 \mathrm{I}=\mathrm{III} 5$ \\
\hline I I $=$ I I I & $53=\mathrm{I} 53$ & $92=$ III 6 \\
\hline $\mathrm{I} 2=\mathrm{I} \mathrm{I} 2$ & $54=\mathrm{I} 54$ & $93=\mathrm{III}_{7}$ \\
\hline $\mathrm{I}_{3}=\mathrm{I}_{\mathrm{I}} 3$ & $55=\mathrm{I} 55$ & $94=$ III 8 \\
\hline $\mathrm{I} 4=\mathrm{I} \mathrm{I} 4$ & $56=I 56$ & $95=$ III 9 \\
\hline Is $=$ I Is & $57=\mathrm{I} 57$ & $96=$ III 10 \\
\hline $\mathrm{I} 6=\mathrm{I} \mathrm{I} 6$ & $-\quad I 57^{a}$ & $97=$ III I I \\
\hline $\mathrm{I} 7=\mathrm{I} \mathrm{I7}$ & & $98=$ III $\mathrm{I} 2$ \\
\hline I $8=\mathrm{I}$ I 8 & $58=$ II I & $99=\mathrm{III} \mathrm{I}_{3}$ \\
\hline $19=$ I 19 & $59=\mathrm{II} 2$ & $\mathrm{I} O 0=\mathrm{III} \mathrm{I} 4$ \\
\hline $20=$ I 20 & $60=\mathrm{II}_{3}$ & $\mathrm{IOI}=\mathrm{III} \mathrm{I} 5$ \\
\hline $2 \mathrm{I}=\mathrm{I} 2 \mathrm{I}$ & $6 \mathrm{I}=\mathrm{II} 4$ & $\mathrm{I} 02=\mathrm{III} \mathrm{I} 6$ \\
\hline $22=\mathrm{I} 22$ & $62=$ II 5 & $\mathrm{I} \circ 3=\mathrm{III}$ I 7 \\
\hline $23=\mathrm{I} 23$ & $63=$ II 6 & $\mathrm{I} O 4=\mathrm{III}$ I 8 \\
\hline $24=\mathrm{I} 24$ & $64=\mathrm{II}_{7}$ & I05 $=$ III I 9 \\
\hline $25=\mathrm{I} 25$ & $65=\mathrm{II} 8$ & $106=$ III 20 \\
\hline $26=$ I 26 & $66=$ II 9 & $107=$ III $2 \mathrm{I}$ \\
\hline $27=\mathrm{I} 27$ & $67=$ II 10 & $108=$ III 22 \\
\hline $28=\mathrm{I} 28$ & $68=$ II I I & $109=\mathrm{III} 23+\mathrm{VI}_{\mathrm{I}}$ \\
\hline $29=$ I 29 & $69=$ II $I_{2}$ & I IO $=$ III $24+$ VI 2 \\
\hline $30=I_{30}$ & $70=$ II I 3 & $\mathrm{III}=\mathrm{III} 25+\mathrm{VI} 3$ \\
\hline $3 \mathrm{I}=\mathrm{I} 3 \mathrm{I}$ & $7 \mathrm{I}=\mathrm{II} \mathrm{I} 4$ & I $\mathrm{I}_{2}=\mathrm{III} 26+\mathrm{VI} 4$ \\
\hline $32=\mathrm{I} 32$ & $72=$ II Is & $\mathrm{II}_{3}=\mathrm{III} 27+\mathrm{VI} 5$ \\
\hline $33=\mathrm{I} 33$ & $73=\mathrm{II} \mathrm{I} 6$ & II $4=$ III $28+$ VI 6 \\
\hline $34=\mathrm{I} 34$ & $74=\mathrm{II} \quad \mathrm{I} 7$ & II $5=$ III $29+$ VI 7 \\
\hline $35=\mathrm{I} 35$ & $75=\mathrm{II}$ I 8 & II $6=\mathrm{III} 30+\mathrm{VI} 8$ \\
\hline $36=I_{3} 6$ & $76=$ II I9 & $\mathrm{II}_{7}=\mathrm{III} 3 \mathrm{I}+\mathrm{VI} 9$ \\
\hline $37=\mathrm{I} 37$ & $77=$ II 20 & $\mathrm{II}_{\mathrm{I}}=\mathrm{III} 32+\mathrm{VI} \mathrm{IO}_{\mathrm{I}}$ \\
\hline $38=\mathrm{I} 38$ & $78=$ II $2 \mathrm{I}$ & $\mathrm{II}_{9}=\mathrm{III} 33+\mathrm{VI}$ II \\
\hline $39=$ I 39 & $79=$ II 22 & $\mathrm{I}_{20}=\mathrm{III}_{34}+\mathrm{VI}_{\mathrm{I}}$ \\
\hline $40=I_{40}$ & $80=$ II 23 & $\mathrm{I}_{2} \mathrm{I}=\mathrm{III} 35+\mathrm{VI}_{\mathrm{I}}$ \\
\hline $4 \mathrm{I}=\mathrm{I} 4 \mathrm{I}$ & $8 \mathrm{I}=\mathrm{II} 24$ & $\mathrm{I}_{22}=\mathrm{III}_{3} 6+\mathrm{VII}_{4}$ \\
\hline $42=I_{42}$ & $82=$ II 25 & I $23=$ III 37 \\
\hline
\end{tabular}


Konkordanz zur Inschrift der Acta Severiana

\begin{tabular}{|c|c|}
\hline $\mathrm{I} 24=\mathrm{III} 38$ & $\mathrm{I} 72=\mathrm{III} 88$ \\
\hline $\mathrm{I} 25=\mathrm{III} 39$ & I73 = III 89 \\
\hline I $26=$ III 40 & $\mathrm{I} 74=\mathrm{III} 90$ \\
\hline $\mathrm{I} 27=\mathrm{III} 4 \mathrm{I}$ & $\mathrm{I} 75=\mathrm{III} 9 \mathrm{I}$ \\
\hline I $28=$ III 42 & \\
\hline I $29=$ III 43 & $\mathrm{I} 76=\mathrm{IV}$ I \\
\hline I $30=$ III 44 & $\mathrm{I} 77=\mathrm{IV} 2$ \\
\hline $\mathrm{I}_{3} \mathrm{I}=\mathrm{III} 45$ & $\mathrm{I}_{7} 8=\mathrm{IV}_{3}$ \\
\hline $\mathrm{I} 32=$ III 46 & $\mathrm{I} 79=\mathrm{IV}_{4}$ \\
\hline $\mathrm{I} 33=\mathrm{III} 47$ & $\mathrm{I} 80=\mathrm{IV} 5$ \\
\hline $\mathrm{I} 34=\mathrm{III}{ }_{4} 8$ & $\mathrm{I} 8 \mathrm{I}=\mathrm{IV} 6$ \\
\hline I $35=$ III 49 & $\mathrm{I}_{82}=\mathrm{IV} 7$ \\
\hline I $36=$ III 50 & $\mathrm{I}_{3}=\mathrm{IV} 8$ \\
\hline $\mathrm{I} 37=\mathrm{III} 5 \mathrm{I}$ & $\mathrm{I} 84=\mathrm{IV} 9$ \\
\hline I $38=$ III 52 & I $85=$ IV Io \\
\hline I $39=$ III 53 & I $86=$ IV I I \\
\hline $\mathrm{I} 40=$ III 54 & I $87=$ IV I 2 \\
\hline $\mathrm{I} 4 \mathrm{I}=\mathrm{III} 55$ & $\mathrm{I} 88=\mathrm{IV}$ I 3 \\
\hline $\mathrm{I}_{42}=$ III 56 & $\mathrm{I} 89=\mathrm{IV} \mathrm{I} 4$ \\
\hline $\mathrm{I} 43=\mathrm{III} 57$ & $\mathrm{I} 90=\mathrm{IV}$ I 5 \\
\hline $\mathrm{I} 44=\mathrm{III} 58$ & I9I $=$ IV I6 \\
\hline $\mathrm{I} 45=$ III 59 & $\mathrm{I} 92=\mathrm{IV}$ I7 \\
\hline $\mathrm{I}_{4} 6=\mathrm{III} 60$ & $\mathrm{I} 93=\mathrm{IV}$ I 8 \\
\hline $\mathrm{I} 47=\mathrm{III} 6 \mathrm{I}$ & I94 = IV I9 \\
\hline $\mathrm{I} 48=\mathrm{III} 62$ & $195=I V 20$ \\
\hline I $49=$ III 63 & I96 = IV 2I \\
\hline I $50=$ III 64 & $197=$ IV 22 \\
\hline $\mathrm{I} 5 \mathrm{I}=\mathrm{III} 65$ & $198=$ IV 23 \\
\hline I $52=$ III 66 & $\mathrm{I} 99=\mathrm{V}^{\mathrm{a}} 23^{\mathrm{a}}+\mathrm{V}_{\mathrm{I}}$ \\
\hline I $53=$ III 67 & $200=V^{a} 24+V_{2}$ \\
\hline I $54=$ III 68 & $20 \mathrm{I}=\mathrm{V}^{\mathrm{a}} 25+\mathrm{V} 3$ \\
\hline I $55=$ III 69 & $202=\mathrm{V}^{\mathrm{a}} 26+\mathrm{V}_{4}$ \\
\hline I $56=$ III 70 & $203=V^{a} 27+V_{5}$ \\
\hline $\mathrm{I} 57=\mathrm{III} 7 \mathrm{I}$ & $204=V^{a} 28+V 6$ \\
\hline I $58=$ III 72 & $205=V^{a} 29+V 7$ \\
\hline $\mathrm{I} 59=\mathrm{III} 73$ & $206=\mathrm{V}^{\mathrm{a}} 30+\mathrm{V} 8$ \\
\hline $\mathrm{I} 60=\mathrm{III} 74$ & $207=\mathrm{V}^{\mathrm{a}} 3 \mathrm{I}+\mathrm{V} 9$ \\
\hline $\mathrm{I} 6 \mathrm{I}=\mathrm{III} 75$ & $208=\mathrm{V}^{\mathrm{a}} 32+\mathrm{V}$ I0 \\
\hline $\mathrm{I} 62=\mathrm{III} 76$ & $209=\mathrm{V}^{\mathrm{a}} 33+\mathrm{V}$ I I \\
\hline I63 = III 77 & $2 \mathrm{ro}=\mathrm{V}^{\mathrm{a}} 34$ \\
\hline $\mathrm{I} 64=\mathrm{III} 78$ & $2 \mathrm{II}=\mathrm{V}^{\mathrm{a}} 35$ \\
\hline${ }^{165}=$ III 79 & $2 \mathrm{I}_{2}=\mathrm{V}^{\mathrm{a}} 36$ \\
\hline $\mathrm{I} 66=\mathrm{III} 80$ & $2 \mathrm{I}_{3}=\mathrm{V}^{\mathrm{a}} 37$ \\
\hline $\mathrm{I} 67=\mathrm{III} 8 \mathrm{I}$ & $2 \mathrm{I} 4=\mathrm{V}^{\mathrm{a}} 3^{8}$ \\
\hline $\mathrm{I} 68=\mathrm{III} 82$ & $2 \mathrm{I} 5=\mathrm{V}^{\mathrm{a}} 39$ \\
\hline I69 $=$ III 83 & $2 \mathrm{I} 6=\mathrm{V}^{\mathrm{a}} 4 \mathrm{O}$ \\
\hline $\mathrm{I} 70=\mathrm{III} 84$ & $2 \mathrm{I} 7=\mathrm{V}^{\mathrm{a}} 4 \mathrm{I}$ \\
\hline $\mathrm{I} 7 \mathrm{I}=\mathrm{III} 85$ & $2 \mathrm{I} 8=\mathrm{V}^{\mathrm{a}} 42$ \\
\hline$-\quad$ III 86 & $2 \mathrm{I} 9=\mathrm{V}^{\mathrm{a}} 43$ \\
\hline $\begin{array}{lll}- & \text { III } 87\end{array}$ & $220=\mathrm{V}^{\mathrm{a}} 44$ \\
\hline
\end{tabular}


$270=$ VIII $^{\mathrm{a}} 2$

$27 \mathrm{I}=$ VIII $^{\mathrm{a}} 3$

$272=$ VIIII $^{\mathrm{a}} 4$

$273=$ VIII $^{a} 5$

$274=$ VIII $^{\mathrm{a}} 6$

$275=$ VIII $^{\mathrm{a}} 7$

$276=$ VIII $^{\mathrm{a}} 8$

$277=$ VIII $^{\mathrm{a}} 9$

$278=$ VIII $^{\mathrm{a}}$ I0

$279=$ VIII $^{\text {a }}$ I I

$280=$ VIII $^{\mathrm{a}} \mathrm{I} 2$

$28 \mathrm{I}=\mathrm{VIII}^{\mathrm{a}} \mathrm{I} 3$

$282=$ VIII $^{\mathrm{a}} \mathrm{I} 4$

$283=$ VIII $^{\text {a }}$ Is

$284=$ VIII $^{\mathrm{a}} \mathrm{I} 6$

$285=$ VIII $^{\mathrm{a}} \mathrm{I} 7$

$286=\mathrm{VIII}^{\mathrm{a}}$ I 8
$287=\mathrm{VIII}^{\mathrm{a}} \mathrm{I} 9$

$288=$ VIII $^{\mathrm{a}} 20$

$289=$ VIII $^{\mathrm{a}} 2 \mathrm{I}$

$290=$ VIII $^{\mathrm{a}} 22$

$29 \mathrm{I}=\mathrm{VIII}^{\mathrm{a}} 23$

$292=$ VIII $^{a} 24$

$293=$ VIII $^{\mathrm{a}} 25$

$294=$ VIII $^{\mathrm{a}} 26$

$295=$ VIII $^{\mathrm{a}} 27$

$296=$ VIII $^{\mathrm{a}} 28$

$297=$ VIII $^{\mathrm{a}} 29$

$298=$ VIII $^{\mathrm{a}} 30$

$299=$ VIII $^{\mathrm{b}} \mathrm{I}$

$300=$ VIII $^{\mathrm{b}} 2$

$30 \mathrm{I}=\mathrm{VIII}^{\mathrm{b}} 3$

$302=$ VIII $^{\mathrm{b}} 4$

$$
\begin{aligned}
& 303=\text { VIII }^{\mathrm{b}} 5 \\
& 304=\text { VIII }^{\mathrm{b}} 6 \\
& 305=\text { VIII }^{\mathrm{b}} 7 \\
& 306=\text { IX I } \\
& 307=\text { IX } 2 \\
& 308=\text { IX }_{3} \\
& 309=\text { IX }_{4} \\
& 310=\text { IX 5 } \\
& 3 \mathrm{II}=\mathrm{IX} 6 \\
& 3 \mathrm{I} 2=\mathrm{IX} 7 \\
& 3 \mathrm{I} 3=\mathrm{IX} 8 \\
& 3 \mathrm{I} 4=\text { IX } 9 \\
& 315=\text { IX } 10 \\
& 3 \mathrm{I} 6=\mathrm{IX} \text { I I } \\
& 3 \mathrm{I} 7=\mathrm{IX} \mathrm{I} 2
\end{aligned}
$$

